\title{
Renormalization group of probabilistic cellular automata with one absorbing state
}

\author{
M. J. de Oliveira and J. E. Satulovsky \\ Universidade de São Paulo, \\ Instituto de Física, Caixa Postal 66318, \\ 05315-970 São Paulo, SP, Brazil
}

(November 8, 2018)

\begin{abstract}
We apply a recently proposed dynamically driven renormalization group scheme to probabilistic cellular automata having one absorbing state. We have found just one unstable fixed point with one relevant direction. In the limit of small transition probability one of the cellular automata reduces to the contact process revealing that the cellular automata are in the same universality class as that process, as expected. Better numerical results are obtained as the approximations for the stationary distribution are improved.
\end{abstract}

\section{INTRODUCTION}

Recently, a general dynamical renormalization group (RG) scheme [1] has been proposed in order to treat nonequilibrium critical phenomena. The method, called Dynamically Driven Renormalization Group (DDRG), has been applied to self-organized critical phenomena, specifically to critical height sandpile models [2] and forest fire models [3]. Its phenomenological approach takes into account the nature of self-organized systems through an attractive fixed point. The scheme also provides numerical values for the critical exponents which are close to the ones obtained using computer simulations.

The scheme consists in coupling a real space RG scheme to a stationary condition that drives the RG group equations through the parameter space. The stationary conditions, involving the stationary distribution, must always be approximated. While the authors used a stationary probability distribution that neglects any correlation among different sites, they have mentioned [1] the fact that using more refined approximations should improve the values of the critical exponents.

While the DDRG scheme is quite general, selforganized critical systems are special since they have a well defined time scale separation (dissipation events being instantaneous with respect to the driving). This prevents proliferation effects in the real space $\mathrm{RG}$, making the calculation of critical exponents easier.

In this work, we implemented the DDRG scheme to another class of systems namely the class of driven diffusive systems with an absorbing state (the vacuum state). Due to the presence of one absorbing state this class of systems is in the same universality as the directed percolation model [4] [5]. More precisely we have considered one-dimensional probabilistic cellular automata with one absorbing state. We have treated two types of models. One of them is a two-state probabilistic cellular automaton (that can be interpreted as directed percolation in two dimensions) that includes, in the limit of small transition probabilities, as particular cases, continuous-time processes with one absorbing state such as the contact model [6] and others [7]. The other one is a four-state probabilistic cellular automaton that includes the model introduced by Grassberger and de la Torre [8] as a particular case.

The appropriate real space $\mathrm{RG}$ parameter space for these nonequilibrium models is the space of the transition probabilities, instead of being the space of coupling constants as is the case of equilibrium models, defined by a Hamiltonian. In the case of the models studied here, with one absorbing state, the RG should be appropriate to preserve the vacuum state along the $R G$ trajectory in this parameter space. By using a block renormalization to treat properly the absorbing state we have figured the value of the critical exponent corresponding to the divergence of the spatial correlation, $\nu_{\perp}$, using three different approximations which consider correlations among clusters up to 1, 3, and 5 neighboring sites in the lattice, respectively. Our best calculations give $\nu_{\perp}=1.04 \pm 0.02$ which is rather close to the one obtained from numerical simulations reported in [8] namely $\nu_{\perp}=1.067 \pm 0.005$.

\section{TWO-STATE MODEL}

The first model is a one-dimensional cellular automaton with just two state per site. Each site can be either vacant, $\sigma_{i}=0$, or occupied by a particle, $\sigma_{i}=1$. At each time step the state of a certain site will depend only on the previous states of that same site and its nearest neighbors. We consider the most general transition probabilities that are homogeneous and symmetric in space. The transition probability $W\left(\sigma \mid \sigma^{\prime}\right)$ from state $\sigma^{\prime}=\left(\sigma_{1}^{\prime}, \sigma_{2}^{\prime}, \cdots, \sigma_{N}^{\prime}\right)$ to state $\sigma=\left(\sigma_{1}, \sigma_{2}, \cdots, \sigma_{N}\right)$ will be given by the product

$$
W\left(\sigma \mid \sigma^{\prime}\right)=\prod_{i=1}^{N} w\left(\sigma_{i} \mid \sigma_{i-1}^{\prime}, \sigma_{i}^{\prime}, \sigma_{i+1}^{\prime}\right)
$$

where $N$ is the number of sites and $w\left(\sigma_{i} \mid \sigma_{i-1}^{\prime}, \sigma_{i}^{\prime}, \sigma_{i+1}^{\prime}\right)$ is the one-site transition probability given by the rules

\begin{tabular}{|c|c|c|c|c|c|c|c|c|}
\hline$w$ & 000 & 001 & 100 & 101 & 010 & 011 & 110 & 111 \\
\hline 0 & 1 & $1-p_{1}$ & $1-p_{1}$ & $1-p_{2}$ & $p_{3}$ & $p_{4}$ & $p_{4}$ & $p_{5}$ \\
\hline 1 & 0 & $p_{1}$ & $p_{1}$ & $p_{2}$ & $1-p_{3}$ & $1-p_{4}$ & $1-p_{4}$ & $1-p_{5}$ \\
\hline
\end{tabular}


The rule $w(0 \mid 000)=1$ implies that the vacuum state is indeed an absorbing state. When $p_{5}=p_{4}=p_{3}$ we say that the annihilation of particles is spontaneous. Suppose moreover that $p_{2}=2 p_{1}$ and that the parameters $p_{1}$ and $p_{3}$ are very small. In this case the system remains most of the time in its previous state. We expect, therefore, that the properties of the present two-state model, in the limit $p_{1} \rightarrow 0$ and $p_{3} \rightarrow 0$, with the ratio $p_{1} / p_{3}=\lambda$ fixed, be identical to the contact process with a catalytic transition rate equal to $\lambda$ and a transition rate for spontaneous annihilation equal to unity. If $p_{2}=p_{1}$, and taking the same limit, the properties will be identical to a model introduced by Dickman (Model A) [7].

The model is represented by a set of five parameters, $p_{1}, p_{2}, p_{3}, p_{4}$, and $p_{5}$, which constitutes, as we shall see, the RG parameter space. The RG scheme will be constructed in a way that the RG trajectory will be confined to this space.

\section{FOUR-STATE MODEL}

Grassberger and the la Torre model [8] is defined as follows. Each site of a one-dimensional lattice is either occupied by one particle or it is void. At a certain time step the state of the system may be defined by the vector $\sigma=\left(\sigma_{1}, \sigma_{2}, \sigma_{3}, \cdots, \sigma_{N}\right)$ where $\sigma_{i}=0$ or 1 according whether the site $i$ is vacant or occupied. In each time step, all sites are updated in two stages.

1) In the first stage each particle is spontaneously annihilated with probability $c$.

2) In the second stage every surviving particle will generate, with probability $p$, a new (unique) particle, which will be placed in one of its nearest neighboring sites, randomly chosen. In other words, for each site with a particle, we chose a neighboring site with probability $p / 2$. If the site was originally void it becomes occupied, and if it was occupied it remains as such. We have modified slightly the original model by introducing the parameter $p$. The original model of Grassberger and de la Torre is recast when $p=1$.

Defined in this way, the transition probability $W\left(\sigma \mid \sigma^{\prime}\right)$ from a state $\sigma^{\prime}$, to another state $\sigma$, can not be written as a product of independent transition probabilities associated to each site $w\left(\sigma_{i} \mid \sigma_{i-1}^{\prime}, \sigma_{i}^{\prime}, \sigma_{i+1}^{\prime}\right)$, as in ordinary cellular automaton. However, if we enlarge the number of states in each site by introducing three types of particle, then it is possible to map the model into a four-state cellular automata. This mapping is outlined in Appendix A.

The four-state probabilistic cellular automaton equivalent to Grassberger and de la Torre model is defined as follows. Each site of a one-dimensional lattice can be either empty $(E), \sigma_{i}=0$, or occupied by a neutral particle $(N), \sigma_{i}=1$, or by a rightist particle $(R), \sigma_{i}=2$, or by a leftist particle $(L), \sigma_{i}=3$. At each time step, every site of the lattice is independently updated according to the rules.

1) If the site is occupied by one particle of any type $N, R$, or $L$, then one out of four possible events will take place.

a) The particle is annihilated, that is, the site becomes empty, with probability $c$, or

b) becomes a particle of type $N$ with probability $a$, or

c) becomes a particle of type $R$ with probability $b / 2$, or

d) becomes a particle of type $L$ with probability $b / 2$.

Here $a=(1-c)(1-p)$ and $b=(1-c) p$.

2) In case the site is empty (state $E$ ) one has to look to its neighboring sites.

a) If its left neighbor is type $R$ or its right neighbor is of type $L$, the site remains as $E$ with probability $c$, becomes $N$ with probability $a$, becomes $R$ with probability $b / 2$, or becomes $L$ with probability $b / 2$.

b) If on the contrary, its left nearest neighbor is not a particle of type $R$ and is right nearest neighbor is not of type $L$, the site remains vacant. This last rule implies that the state with all sites empty is an absorbing state.

Transition probability $W\left(\sigma \mid \sigma^{\prime}\right)$ from state $\sigma^{\prime}=$ $\left(\sigma_{1}^{\prime}, \sigma_{2}^{\prime}, \cdots, \sigma_{N}^{\prime}\right)$ to state $\sigma=\left(\sigma_{1}, \sigma_{2}, \cdots, \sigma_{N}\right)$ can be written as the product

$$
W\left(\sigma \mid \sigma^{\prime}\right)=\prod_{i=1}^{N} w\left(\sigma_{i} \mid \sigma_{i-1}^{\prime}, \sigma_{i}^{\prime}, \sigma_{i+1}^{\prime}\right)
$$

where $N$ is the number of sites of the lattice. The onesite transition probability $w\left(\sigma_{i} \mid \sigma_{i-1}^{\prime}, \sigma_{i}^{\prime}, \sigma_{i+1}^{\prime}\right)$ is written down in the Appendix A.

In order to apply the RG scheme we enlarge the space of parameters but preserve the existence of the absorbing state. Demanding besides that the rules should be homogeneous in space and invariant by exchanging the states $R$ and $L$, the transition probability $w\left(\sigma_{i} \mid \sigma_{i-1}^{\prime}, \sigma_{i}^{\prime}, \sigma_{i+1}^{\prime}\right)$ will be defined in the most general way by 59 parameters. We call such a probabilistic cellular automaton the four-state model. 


\section{RENORMALIZATION SCHEME}

Here we use a real space RG scheme [9-11] which renormalizes the transition probability $W$. The succession of RG transformations corresponds to a trajectory in the space spanned by the parameters that defines $W$. The scheme we use is an implementation of the DDRG [1] and is accomplished by transforming cells of $b$ sites into a cell of just one site. To treat the vacuum state properly any cell with at least one particle renormalizes into an occupied site. Only cells with no particles renormalizes into a vacant site.

Let $\mathcal{R}(\tau \mid \sigma)$ be a condition probability of state $\tau$ given the state $\sigma$ with the following properties

$$
\mathcal{R}(\tau \mid \sigma) \geq 0, \quad \quad \sum_{\tau} \mathcal{R}(\tau \mid \sigma)=1
$$

The vector $\sigma=\left(\sigma_{1}, \sigma_{2}, \cdots, \sigma_{N}\right)$ represents the state of a system with $N$ degrees of freedom and the vector $\tau=\left(\tau_{1}, \tau_{2}, \cdots, \tau_{N^{\prime}}\right)$ represents the state of the renormalized system with $N^{\prime}=N / b$ degrees of freedom, where $b$ is the size of the renormalization block.

Let $P_{n}\left(\sigma, \sigma^{\prime}\right)$ be the probability of occurrence of state $\sigma^{\prime}$ at a given time and state $\sigma$ at $n$ time steps later, that is

$$
P_{n}\left(\sigma, \sigma^{\prime}\right)=W^{n}\left(\sigma \mid \sigma^{\prime}\right) P\left(\sigma^{\prime}\right)
$$

where $P(\sigma)$ is the stationary probability distribution which satisfies the equation

$$
P(\sigma)=\sum_{\sigma^{\prime}} W^{n}\left(\sigma \mid \sigma^{\prime}\right) P\left(\sigma^{\prime}\right)
$$

for any value of $n$, where $W^{n}\left(\sigma \mid \sigma^{\prime}\right)$ is the transition probability from state $\sigma^{\prime}$ to state $\sigma$ in $n$ time steps. Similarly, for the renormalized system, let $\widetilde{P}\left(\tau, \tau^{\prime}\right)$ be the probability of occurrence of state $\tau^{\prime}$ at a given time and state $\tau$ at one time step later. The RG transformation are obtained by demanding that 9

$$
\widetilde{P}\left(\tau, \tau^{\prime}\right)=\sum_{\sigma} \sum_{\sigma^{\prime}} \mathcal{R}(\tau \mid \sigma) \mathcal{R}\left(\tau^{\prime} \mid \sigma^{\prime}\right) P_{n}\left(\sigma, \sigma^{\prime}\right)
$$

from which follows

$$
\widetilde{P}\left(\tau^{\prime}\right)=\sum_{\sigma^{\prime}} \mathcal{R}\left(\tau^{\prime} \mid \sigma^{\prime}\right) P\left(\sigma^{\prime}\right)
$$

since

$$
\widetilde{P}\left(\tau^{\prime}\right)=\sum_{\tau} \widetilde{P}\left(\tau, \tau^{\prime}\right)
$$

To get the desired renormalized transition probability $\widetilde{W}\left(\tau \mid \tau^{\prime}\right)$ we use

$$
\widetilde{W}\left(\tau \mid \tau^{\prime}\right)=\frac{\widetilde{P}\left(\tau, \tau^{\prime}\right)}{\widetilde{P}\left(\tau^{\prime}\right)}
$$

and equations (5), (7), and (8). We obtain [1]

$$
\widetilde{W}\left(\tau \mid \tau^{\prime}\right)=\frac{\sum_{\sigma} \sum_{\sigma^{\prime}} \mathcal{R}(\tau \mid \sigma) \mathcal{R}\left(\tau^{\prime} \mid \sigma^{\prime}\right) W^{n}\left(\sigma \mid \sigma^{\prime}\right) P\left(\sigma^{\prime}\right)}{\sum_{\sigma^{\prime}} \mathcal{R}\left(\tau^{\prime} \mid \sigma^{\prime}\right) P\left(\sigma^{\prime}\right)}
$$

This equation, however, is not properly a transformation between the transition probabilities $W$ and $\widetilde{W}$, since the yet unknown stationary probability $P(\sigma)$ appears in the right hand side of equation (11). However, if we use the balance equation (6) for $P(\sigma)$ then a closure condition for the renormalization group is obtained. The closure condition plays the role of the driving condition that forces the system to be in the stationary state at each step of the transformation. In this sense the present DDRG may be thought of as a renormalization of the stationary state.

At each state of the transformation the transition probability $W$ always describes an irreversible process so that the corresponding stationary solution $P(\sigma)$ obtained from the closure condition (6) will not be related, a priori, to a Hamiltonian, that would be the case if the process obeyed detailed balance. In this way the present RG scheme is distinct from the ordinary real space RG used in equilibrium systems in which the parameters of the Hamiltonian are renormalized.

The closure relation (6), however, cannot actually be solved so that approximations should be used. Here we have used three different approximations which consider correlations among clusters up to 1,3 , and 5 neighboring sites. Equation (11) together with a given approximation provides then a well defined RG transformation $W \rightarrow \widetilde{W}$.

Assuming that the renormalized transition probability can also be written as a product of independent transition probabilities, that is,

$$
\widetilde{W}\left(\tau \mid \tau^{\prime}\right)=\prod_{k=1}^{N^{\prime}} \widetilde{w}\left(\tau_{k} \mid \tau_{k-1}^{\prime}, \tau_{k}^{\prime}, \tau_{k+1}^{\prime}\right)
$$

one can write the RG equations that relates the old onesite transition probability $w\left(\sigma_{i} \mid \sigma_{i-1}^{\prime}, \sigma_{i}^{\prime}, \sigma_{i+1}^{\prime}\right)$ to the new one $\widetilde{w}\left(\tau_{k} \mid \tau_{k-1}^{\prime}, \tau_{k}^{\prime}, \tau_{k+1}^{\prime}\right)$.

We have used only renormalization cells with size $b=2$ and chosen $\mathcal{R}$ in the form

$$
\mathcal{R}(\tau \mid \sigma)=\prod_{k=1}^{N / 2} R\left(\tau_{k} \mid \sigma_{2 k-1}, \sigma_{2 k}\right)
$$

with

$$
R\left(\tau_{k} \mid \sigma_{2 k-1}, \sigma_{2 k}\right) \geq 0, \text { and } \quad \sum_{\tau_{k}} R\left(\tau_{k} \mid \sigma_{2 k-1}, \sigma_{2 k}\right)=1
$$

To preserve the absorbing nature of the vacuum state we have chosen $R$ with the properties

$$
R(0 \mid 0,0)=1
$$


and

$$
R\left(0 \mid \sigma_{2 k-1}, \sigma_{2 k}\right)=0
$$

whenever $\sigma_{2 k-1} \neq 0$, or $\sigma_{2 k} \neq 0$.

For the four-site model we used the following values

$$
\begin{array}{cccc}
R(0 \mid 00)=1 & R(1 \mid 01)=1 & R(1 \mid 10)=1 & R(1 \mid 11)=1 \\
R(2 \mid 02)=1 & R(1 \mid 03)=1 & R(1 \mid 20)=1 & R(3 \mid 30)=1 \\
R(2 \mid 12)=1 & R(1 \mid 13)=1 & R(1 \mid 21)=1 & R(3 \mid 31)=1 \\
R(2 \mid 32)=1 / 2 & R(3 \mid 32)=1 / 2 & R(2 \mid 22)=1 & R(1 \mid 23)=1
\end{array}
$$

The other matrix elements of $R$ are zero. The first matrix element has been chosen in order to preserve the absorbing nature of the vacuum state and the rest of the elements where assigned bearing in mind the physical picture that an $R$ particle will give rise to a new particle to the right in the next time step, while an $L$ particle will generate a new particle to the left ( $N$ particles will not generate new particles).

\section{RENORMALIZATION ALGORITHM}

The temporal coarse graining will be done using two time steps that is $n=2$. Using the equations of the previous section we can write down the equation that relates $w$ to $\widetilde{w}$ for the case $n=2$ (see figure 1 ).

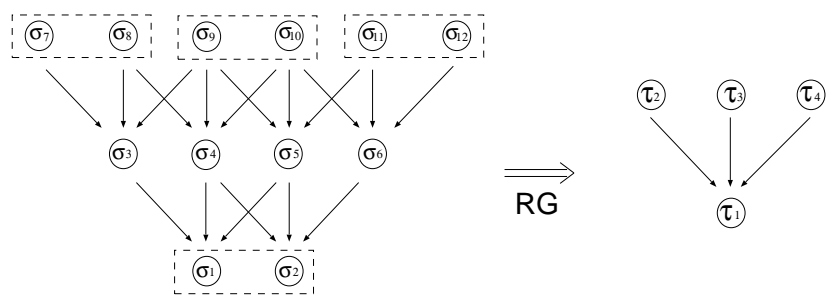

FIG. 1. Diagram showing the blocking scheme procedure. Numbers correspond to the indexes used in equation (17).

It is given by

$$
\begin{aligned}
& \widetilde{w}\left(\tau_{1} \mid \tau_{2}, \tau_{3}, \tau_{4}\right)=\sum_{\sigma_{1} \sigma_{2} \sigma_{7} \cdots \sigma_{12}} R\left(\tau_{1} \mid \sigma_{1} \sigma_{2}\right) \times \\
& \quad T\left(\sigma_{1}, \sigma_{2} \mid \sigma_{7}, \cdots, \sigma_{12}\right) \rho\left(\sigma_{7}, \cdots, \sigma_{12} \mid \tau_{2}, \tau_{3}, \tau_{4}\right),
\end{aligned}
$$

where

$$
\begin{aligned}
& T\left(\sigma_{1} \sigma_{2} \mid \sigma_{7}, \sigma_{8}, \sigma_{9}, \sigma_{10}, \sigma_{11}, \sigma_{12}\right)= \\
& \sum_{\sigma_{3} \sigma_{4} \sigma_{5} \sigma_{6}} w\left(\sigma_{1} \mid \sigma_{3}, \sigma_{4}, \sigma_{5}\right) w\left(\sigma_{2} \mid \sigma_{4}, \sigma_{5}, \sigma_{6}\right) \times \\
& w\left(\sigma_{3} \mid \sigma_{7}, \sigma_{8}, \sigma_{9}\right) w\left(\sigma_{4} \mid \sigma_{8}, \sigma_{9}, \sigma_{10}\right) \times \\
& w\left(\sigma_{5} \mid \sigma_{9}, \sigma_{10}, \sigma_{11}\right) w\left(\sigma_{6} \mid \sigma_{10}, \sigma_{11}, \sigma_{12}\right),
\end{aligned}
$$

and

$$
\begin{aligned}
& \rho\left(\sigma_{7}, \sigma_{8}, \sigma_{9}, \sigma_{10}, \sigma_{11}, \sigma_{12} \mid \tau_{2}, \tau_{3}, \tau_{4}\right)=\left[\widetilde{P}\left(\tau_{2}, \tau_{3}, \tau_{4}\right)\right]^{-1} \times \\
& \quad R\left(\tau_{2} \mid \sigma_{7}, \sigma_{8}\right) R\left(\tau_{3} \mid \sigma_{9}, \sigma_{10}\right) R\left(\tau_{4} \mid \sigma_{11}, \sigma_{12}\right) \times \\
& \quad P\left(\sigma_{7}, \sigma_{8}, \sigma_{9}, \sigma_{10}, \sigma_{11}, \sigma_{12}\right),
\end{aligned}
$$

where

$$
\begin{array}{r}
\widetilde{P}\left(\tau_{2}, \tau_{3}, \tau_{4}\right)=\sum_{\sigma_{7} \cdots \sigma_{12}} R\left(\tau_{2} \mid \sigma_{7}, \sigma_{8}\right) R\left(\tau_{3} \mid \sigma_{9}, \sigma_{10}\right) \times \\
R\left(\tau_{4} \mid \sigma_{11}, \sigma_{12}\right) P\left(\sigma_{7}, \sigma_{8}, \sigma_{9}, \sigma_{10}, \sigma_{11}, \sigma_{12}\right) .
\end{array}
$$

Here the subscripts refer to the site numbers appearing in figure 1.

In order to solve the system of equations (17-20) one must resort on approximate methods to estimate the stationary weights $P\left(\sigma_{7}, \cdots, \sigma_{12}\right)$. The simplest approximation, sometimes known as simple mean field approximation, consists in neglecting correlations among different sites

$$
P\left(\sigma_{7}, \cdots, \sigma_{12}\right)=\prod_{i=7}^{12} P\left(\sigma_{i}\right)
$$

where $P\left(\sigma_{i}\right)$ is the solution of

$$
P\left(\sigma_{1}\right)=\sum_{\sigma_{2} \sigma_{3} \sigma_{4}} w\left(\sigma_{1} \mid \sigma_{2} \sigma_{3} \sigma_{4}\right) P\left(\sigma_{2}\right) P\left(\sigma_{3}\right) P\left(\sigma_{4}\right) .
$$

However, correlations are actually taken into account in the geometrical aspects of the blocking procedure. In this way, one obtains non classical critical exponents. Given a blocking prescription, the value of the critical exponents should improve as correlations are taken into account into the stationary probability distribution. In order to verify how important the changes will be, we have used three different approximations for the stationary distribution. The first one being (21-22), while the other two are mean field approximations which consider correlations up to clusters of three sites and five sites respectively 12 .

Due to the number of terms involved in equations (17. 20 ) it is not possible to determine analytically the fixed point of the transformation. So, we have performed it numerically, using initial values for the transition probabilities corresponding to the model of interest. In the case of the two-state model we start with $w\left(\sigma_{i} \mid \sigma_{i-1}^{\prime}, \sigma_{i}^{\prime}, \sigma_{i+1}^{\prime}\right)$ given by (2) with $p_{5}=p_{4}=p_{3}$ and $p_{2}=2 p_{1}$. In the case of the four-state model we start with $w\left(\sigma_{i} \mid \sigma_{i-1}^{\prime}, \sigma_{i}^{\prime}, \sigma_{i+1}^{\prime}\right)$ corresponding to the Grassberger and de la Torre model, with $p=1$.

In each iteration of the $\mathrm{RG}$, given the set of parameters $\widetilde{w}$, one has to find the stationary solution for $P\left(\sigma_{7}, \cdots, \sigma_{12}\right)$. This has been done by iterating the time evolution equation for the model (using one of the three approximations) until reaching convergence. For the onesite approximation, given by (22), $10^{4}$ iterations were enough. As approximations are refined equations become highly nonlinear, and for the five-site approximation least $10^{5}$ iterations are needed. 


\section{RESULTS}

For the four-state model, the RG equations behaved in the following way. For small values of $c$, the set of transition matrix elements flows towards an attractive fixed point characterized by $c=0$, and a lattice full of particles. On the contrary, for values of $c$ high enough, the $\mathrm{RG}$ equations are driven to a different attractive fixed point, this time characterized by $c=1$ and a lattice without particles. In this case we used only the one-site approximation and found a critical value of $c$ given by $c_{c r}=0.3568$. Starting around this values the representative point of the parameter set spends a long time near an unstable point before it leaves towards any of the two attractive fixed points. Figure 2 shows a projection of two trajectories in the parameter space in terms of two of these parameters: $w(0 \mid 010)$ and $1-w(0 \mid 101)$.

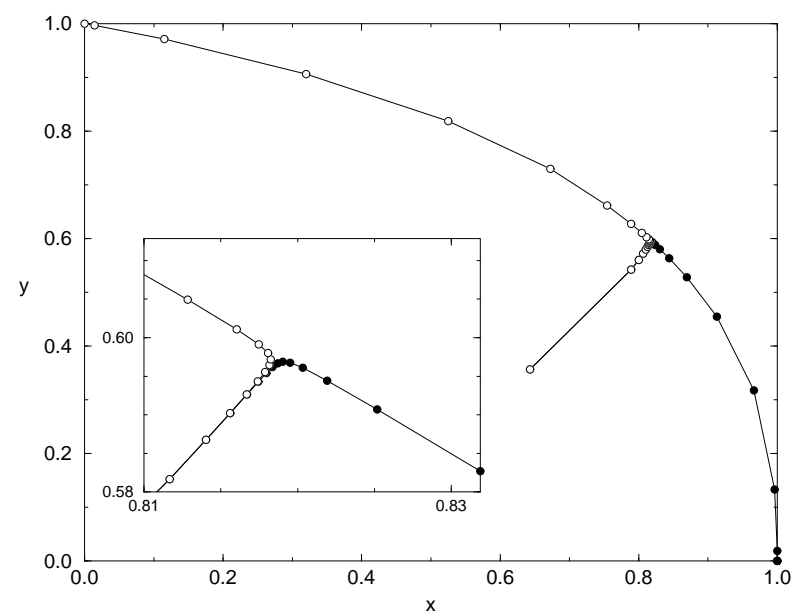

FIG. 2. Projection of two trajectories in the parameter space of the four-state model in terms of two of these parameters: $x=1-\widetilde{w}(0 \mid 101)$ and $y=\widetilde{w}(0 \mid 010)$. Each trajectory reaches a different fixed point. The inset shows an enlargement around the nontrivial fixed point.

In this way, it is found only one relevant parameter. Since we are dealing only with stationary properties of the model it is reasonable to assume that this parameter is associated to the divergence of the spatial correlation length and not to the temporal correlation length. So, figuring the eigenvalue $\Lambda$ associated to that parameter we get $\nu_{\perp}=\ln 2 / \ln \Lambda$. The value measured numerically is $\nu_{\perp}=0.93 \pm 0.005$.

From computer simulations results reported in [8] one can obtain $\nu_{\perp}=1.067 \pm 0.005$, and the critical value of $c$ is $c_{c r}=0.279$. The discrepancy between the two results are mainly due to the poorness of the one-site approximation. By increasing the order of approximation the results gets better as we shall see in the case of the two-site model.

We have corroborated that, as one would expect, the value of $\nu_{\perp}$ for the simpler two-state model and one-site approximation is identical as the one previously found. Since for refined approximations of three and five sites, numerical computations become too imposing, we have used these two better approximations only for the twostate model. The value found using the three-site approximation was $\nu_{\perp}=0.98 \pm 0.01$ whereas the value found in the five-site approximation was $\nu_{\perp}=1.04 \pm 0.02$, which is rather close to the one calculated from numerical simulations. Bellow we show the coordinates of the unstable fixed point for the three approximations, as well as the corresponding value of $\Lambda$

\begin{tabular}{|c|c|c|c|c|c|c|}
\hline appr. & $p_{1}$ & $p_{2}$ & $p_{3}$ & $p_{4}$ & $p_{5}$ & $\Lambda$ \\
\hline 1 & 0.22794 & 0.40381 & 0.26780 & 0.22116 & 0.18245 & 2.105 \\
\hline 3 & 0.12107 & 0.22750 & 0.14761 & 0.07870 & 0.03140 & 2.025 \\
\hline 5 & 0.07107 & 0.16233 & 0.07330 & 0.04783 & 0.01933 & 1.950 \\
\hline
\end{tabular}

Another advantage in using better approximations is the improvements one obtains in the critical values of nonuniversal parameters. We have consider the following initial conditions for the two-state model: $p_{5}=p_{4}=p_{3}$ and $p_{2}=2 p_{1}$. By varying $p_{1}$ and $p_{3}$ we have obtained the critical values $\lambda_{c}$ of the ratio $\lambda=p_{1} / p_{3}$ as shown in the table

\begin{tabular}{|cc|c|c|c|c|}
\hline$p_{1}$ & 1 & 0.1 & 0.01 & 0.001 \\
\hline$\lambda_{c}$ & (1-site app.) & 2.8027 & 2.0833 & 2.0088 & 2.0008 \\
\hline$\lambda_{c}$ & (3-site app.) & 3.2365 & 3.1205 & 3.1703 & 3.1757 \\
\hline$\lambda_{c}$ & (5-site app.) & 3.2555 & 3.1645 & 3.2206 & 3.2300 \\
\hline
\end{tabular}

which, in the limit $p_{1} \rightarrow 0$, should be compared with the critical value of the contact process $\lambda_{c}=3.299$ [13]. The fact that the column associated to $p_{1}=1$ also seems to converge to this value is a mere coincidence.

Reasoning along this line, one may wonder why not to use the stationary distribution directly from a computer simulation of the model. Making large statistics over configurations in the stationary regime, one should be able to accurately estimate the probabilities of the clusters appearing on the left hand side of equations $(19)$ and $(20)$. While the idea is in principle right, one can not overcome in practice the huge amount of time needed to obtain values that are accurate enough. Slight fluctuations in the estimated values will make trajectories randomly shift their destination towards one of the two attractive fixed points, depending on the random seed used in the simulation. This effect takes place even before any trajectory is able to reach the linearized domain of the transformation around the unstable point. A way to decrease fluctuations is increasing the size of the lattice in which one performs the simulation. But the precision one gains does not grow faster than $\sim 1 / \sqrt{N}$, where $N$ is the size of the lattice.

\section{CONCLUSION}

We have applied a real space renormalization group scheme to a class of driven diffusive probabilistic cellular 
automata having one absorbing state. Two models having been considered. One of them is a two-state model that reduces to the contact process in the limit of small transition probabilities. The two-state model can also be interpreted as a generic directed percolation in two dimensions. The other is a four-state models that includes the model introduced by Grassberger and de la Torre in a study related to the contact process. We have found, in the RG space of parameters, just one non-trivial unstable fixed point with one relevant direction. The existence of just this unique fixed point reveals that the probabilistic cellular automata with one absorbing state belongs to the same universality class as the directed percolation and the contact process, as expected.

The implementation of the RG scheme nedeed the calculation of the stationary probability distribution which were performed in several levels of approximations. Increasing the number of cluster size used in the approximation improved results were obtained not only for the critical exponent $\nu_{\perp}$ as well as for the non-universal critical quantities.

\section{APPENDIX A}

The Grassberger and de la Torre model is a stochastic process in which at each time step particles are being created and annihilated. We imagine the process as a sequence os states $A, A^{\prime}, A^{\prime \prime}, A^{\prime \prime \prime}, \cdots$ each one being given by a vector $\eta=\left(\eta_{1}, \eta_{2}, \cdots, \eta_{N}\right)$ where $\eta_{i}=0$ or 1 according whether site $i$ is vacant or occupied by a particle. We may think of each transition, say $A \rightarrow A^{\prime}$, as composed of three stages with two intermediate states $B$ and $C$, to be defined shortly, so that the whole stochastic process corresponds to a sequence $A, B, C, A^{\prime}, B^{\prime}, C^{\prime}, A^{\prime \prime}, B^{\prime \prime}$, $C^{\prime \prime}, A^{\prime \prime \prime}, \cdots$ We will then write the transition probability $W\left(A^{\prime} \mid A\right)$ from state $A$ to $A^{\prime}$ as given by

$$
W\left(A^{\prime} \mid A\right)=\sum_{B} \sum_{C} W_{3}\left(A^{\prime} \mid C\right) W_{2}(C \mid B) W_{1}(B \mid A) .
$$

where $W_{3}\left(A^{\prime} \mid C\right), W_{2}(C \mid B)$, and $W_{1}(B \mid A)$ are the intermediate transition probabilities related to the three stages.

First stage. In the first stage $(A \rightarrow B)$ of the Grassberger and de la Torre model, each particle is annihilated with probability $c$, so that the probability $W_{1}(B \mid A)$ of the transition from $A$ to $B$ is given by

$$
W_{1}(B \mid A)=W_{1}\left(\eta^{\prime \prime} \mid \eta\right)=\prod_{i=1}^{N} w_{1}\left(\eta_{i}^{\prime \prime} \mid \eta_{i}\right),
$$

where

$$
\begin{array}{ll}
w_{1}(0 \mid 1)=c, & w_{1}(1 \mid 1)=1-c \\
w_{1}(1 \mid 0)=0, & w_{1}(0 \mid 0)=1 .
\end{array}
$$

Second stage. In this stage $(B \rightarrow C)$ every particle decides whether it will generate new particles either to the left or to the right in the next step. Each occupied site will be labeled according to its decision. A particle that decides not to generate particles will be labeled by the number 1. A particle that decides to generate another one to the right, will be labeled by the number 2 and a particle that decides to generate a new particle to the left, will be labeled by the number 3 . A state of type $C$ is then defined by the vector $\sigma=\left(\sigma_{1}, \sigma_{2}, \cdots, \sigma_{N}\right)$ where $\sigma_{i}=0,1,2,3$, so that the transition probability $W_{2}(C \mid B)$ of the transition from $B$ to $C$ is given by

$$
W_{2}(C \mid B)=W_{2}\left(\sigma \mid \eta^{\prime \prime}\right)=\prod_{i=1}^{N} w_{2}\left(\sigma_{i} \mid \eta_{i}^{\prime \prime}\right)
$$

where

$$
\begin{array}{ll}
w_{2}(0 \mid 0)=1, & w_{2}(1 \mid 0)=0 \\
w_{2}(2 \mid 0)=0, & w_{2}(3 \mid 0)=0 . \\
w_{2}(0 \mid 1)=0, & w_{2}(1 \mid 1)=1-p, \\
w_{2}(2 \mid 1)=\frac{p}{2}, & w_{2}(3 \mid 1)=\frac{p}{2},
\end{array}
$$

Third stage. In this state $\left(C \rightarrow A^{\prime}\right)$, particles are effectively created. Each occupied site remains occupied. Each vacant site becomes occupied if the site at right (left) is occupied by a particle of type 3 (2).

A configuration of type $A^{\prime}$ is expressed again in terms of the two state variables, $\eta_{i}=0$ or 1 , and the transition probability $W_{2}\left(A^{\prime} \mid C\right)$ of the transition from $C$ to $A^{\prime}$ is given by

$$
W_{3}\left(A^{\prime} \mid C\right)=W_{3}\left(\eta^{\prime} \mid \sigma\right)=\prod_{i=1}^{N} w_{3}\left(\eta_{i}^{\prime} \mid \sigma_{i-1}, \sigma_{i}, \sigma_{i+1}\right),
$$

where the transition probability $w_{3}\left(1 \mid \sigma_{i-1}, \sigma_{i}, \sigma_{i+1}\right)$ to the state $\eta_{i}^{\prime}=1$ is given by

$$
w_{3}\left(1 \mid \sigma_{i-1}, \sigma_{i}, \sigma_{i+1}\right)=1
$$

if $\sigma_{i} \neq 0$, for any value of $\sigma_{i-1}$ and $\sigma_{i+1}$, and

$$
w_{3}\left(1 \mid \sigma_{i-1}, 0, \sigma_{i+1}\right)=1
$$

if $\sigma_{i-1}=2$ or $\sigma_{i+1}=3$. In other cases $w_{3}\left(1 \mid \sigma_{i-1}, \sigma_{i}, \sigma_{i+1}\right)$ vanishes. The transition probability $w_{3}\left(0 \mid \sigma_{i-1}, \sigma_{i}, \sigma_{i+1}\right)$ to the state $\eta_{i}^{\prime}=0$ is just given by

$$
w_{3}\left(0 \mid \sigma_{i-1}, \sigma_{i}, \sigma_{i+1}\right)=1-w_{3}\left(1 \mid \sigma_{i-1}, \sigma_{i}, \sigma_{i+1}\right)
$$

It ease to check that $W\left(A^{\prime} \mid A\right)=W\left(\eta^{\prime} \mid \eta\right)$ cannot be written as product of independent transition probability of each site as in an ordinary cellular automaton. However, the transition probability $W_{c}\left(C^{\prime} \mid C\right)=W_{c}\left(\sigma^{\prime} \mid \sigma\right)$ from state $C$ to state $C^{\prime}$ can. Indeed, from

$$
W_{c}\left(C^{\prime} \mid C\right)=\sum_{B^{\prime}} \sum_{A^{\prime}} W_{2}\left(C^{\prime} \mid B^{\prime}\right) W_{1}\left(B^{\prime} \mid A^{\prime}\right) W_{3}\left(A^{\prime} \mid C\right)
$$


that is from

$$
W_{c}\left(\sigma^{\prime} \mid \sigma\right)=\sum_{\eta^{\prime \prime}} \sum_{\eta^{\prime}} W_{2}\left(\sigma^{\prime} \mid \eta^{\prime \prime}\right) W_{1}\left(\eta^{\prime \prime} \mid \eta^{\prime}\right) W_{3}\left(\eta^{\prime} \mid \sigma\right)
$$

we get

$$
\begin{array}{r}
W_{c}\left(\sigma^{\prime} \mid \sigma\right)=\sum_{\eta^{\prime \prime}} \sum_{\eta^{\prime}} \prod_{i=1}^{N} w_{2}\left(\sigma_{i}^{\prime} \mid \eta_{i}^{\prime \prime}\right) w_{1}\left(\eta_{i}^{\prime \prime} \mid \eta_{i}^{\prime}\right) \times \\
w_{3}\left(\eta_{i}^{\prime} \mid \sigma_{i-1}, \sigma_{i}, \sigma_{i+1}\right)
\end{array}
$$

which can be written in the form

$$
W_{c}\left(\sigma^{\prime} \mid \sigma\right)=\prod_{i=1}^{N} w\left(\sigma_{i}^{\prime} \mid \sigma_{i-1}, \sigma_{i}, \sigma_{i+1}\right)
$$

where

$$
\begin{array}{r}
w\left(\sigma_{i}^{\prime} \mid \sigma_{i-1}, \sigma_{i}, \sigma_{i+1}\right)=\sum_{\eta_{i}^{\prime \prime}} \sum_{\eta_{i}^{\prime}} w_{2}\left(\sigma_{i}^{\prime} \mid \eta_{i}^{\prime \prime}\right) w_{1}\left(\eta_{i}^{\prime \prime} \mid \eta_{i}^{\prime}\right) \times \\
w_{3}\left(\eta_{i}^{\prime} \mid \sigma_{i-1}, \sigma_{i}, \sigma_{i+1}\right)
\end{array}
$$

The Grassberger and de la Torre process can then be viewed as sequence of states $C, C^{\prime}, C^{\prime \prime}, C^{\prime \prime \prime}, \cdots$, each one being given by a vector $\sigma=\left(\sigma_{1}, \sigma_{2}, \cdots, \sigma_{N}\right)$ where $\sigma_{i}=0,1,2$, or 3 according whether site $i$ is either vacant, or occupied by a particle that does not generate another particle (neutral particle), or occupied by a particle that generates another one to the right (a rightist particle) or occupied by a particle that generates another one to the left (a leftist particle). Therefore, it is equivalent to an ordinary four-state cellular automaton whose rules are defined by equations (37), (26), (28), and (30-31. From these equations we may write down the transition probability $w\left(\sigma_{i}^{\prime} \mid \sigma_{i-1}, \sigma_{i}, \sigma_{i+1}\right)$ in the form

$$
\begin{aligned}
& w\left(0 \mid \sigma_{i-1}^{\prime}, \sigma_{i}^{\prime}, \sigma_{i+1}^{\prime}\right)=c, \\
& w\left(1 \mid \sigma_{i-1}^{\prime}, \sigma_{i}^{\prime}, \sigma_{i+1}^{\prime}\right)=a, \\
& w\left(2 \mid \sigma_{i-1}^{\prime}, \sigma_{i}^{\prime}, \sigma_{i+1}^{\prime}\right)=b / 2, \\
& w\left(3 \mid \sigma_{i-1}^{\prime}, \sigma_{i}^{\prime}, \sigma_{i+1}^{\prime}\right)=b / 2,
\end{aligned}
$$

if $\sigma_{i}^{\prime} \neq 0$, independently of the states taken by $\sigma_{i-1}^{\prime}$, and $\sigma_{i+1}^{\prime}$. For the case where $\sigma_{i}^{\prime}=0$, and either $\sigma_{i-1}^{\prime}=2$ or $\sigma_{i+1}^{\prime}=3$, one has

$$
\begin{aligned}
& w\left(0 \mid \sigma_{i-1}^{\prime}, 0, \sigma_{i+1}^{\prime}\right)=c \\
& w\left(1 \mid \sigma_{i-1}^{\prime}, 0, \sigma_{i+1}^{\prime}\right)=a \\
& w\left(2 \mid \sigma_{i-1}^{\prime}, 0, \sigma_{i+1}^{\prime}\right)=b / 2, \\
& w\left(3 \mid \sigma_{i-1}^{\prime}, 0, \sigma_{i+1}^{\prime}\right)=b / 2 .
\end{aligned}
$$

And finally, when $\sigma_{i}^{\prime}=0$, and $\sigma_{i-1}^{\prime} \neq 2$ and $\sigma_{i+1}^{\prime} \neq 3$,

$$
\begin{aligned}
& w\left(0 \mid \sigma_{i-1}^{\prime}, 0, \sigma_{i+1}^{\prime}\right)=1 \\
& w\left(1 \mid \sigma_{i-1}^{\prime}, 0, \sigma_{i+1}^{\prime}\right)=0 \\
& w\left(2 \mid \sigma_{i-1}^{\prime}, 0, \sigma_{i+1}^{\prime}\right)=0 \\
& w\left(3 \mid \sigma_{i-1}^{\prime}, 0, \sigma_{i+1}^{\prime}\right)=0 .
\end{aligned}
$$

The parameters $a$ and $b$ are related to $p$ by $a=(1-$ $p)(1-c)$ and $b=p(1-c)$.

The rule $w(0 \mid 0,0,0)=1$ implies that the state with all sites vacant is indeed an absorbing state.

\section{ACKNOWLEDGMENTS}

J. E. S. would like to acknowledge financial support by FAPESP (Fundação de Amparo à Pesquisa do Estado de São Paulo).

[1] A. Vespignani, S. Zapperi and V. Loreto, Phys. Rev. Lett. 77, 4560 (1996).

[2] A. Vespignani, S. Zapperi and L. Pietronero, Phys. Rev. E 51, 1711 (1995).

[3] V. Loreto, A. Vespignani and S. Zapperi, J. Phys. A 29, 2981 (1996).

[4] H. K. Janssen, Z. Phys. B 42, 151 (1981)

[5] P. Grassberger, Z. Phys. B 47, 365 (1982).

[6] T. M. Liggett, Interacting Particle Systems, SpringerVerlag, New York, 1985.

[7] R. Dickman, Phys. Rev. B 40, 7005 (1989).

[8] P. Grassbeger and A. de la Torre, Ann. Phys. 122, 373 (1979).

[9] G. F. Mazenko, M. J. Nolan and O. T. Valls, Phys. Rev. Lett. 41, 500 (1978).

[10] G. F. Mazenko and O. T. Valls, Phys. Rev. B 31, 1565 (1985).

[11] F. Haake and M. Lewenstein, Phys. Rev. B 27, 5868 (1983).

[12] R. Dickman and T. Tomé, Phys. Rev. A 44, 4833 (1991).

[13] R. C. Brower, M. A. Furman, and M. Moshe, Phys. Lett. B 76, 213 (1978). 